\title{
Optimal sampling site for mucosal candidosis in oral cancer patients is the labial sulcus
}

\author{
Correspondence \\ Riina Rautemaa \\ riina.richardson@helsinki.fi
}

Received 8 March 2006

Accepted 13 July 2006

\author{
Riina Rautemaa, ${ }^{1,2,3}$ Peter Rusanen, ${ }^{1,4}$ Malcolm Richardson ${ }^{1,3}$ \\ and Jukka H. Meurman ${ }^{2,4}$ \\ ${ }^{1}$ Department of Bacteriology and Immunology, Haartman Institute, University of Helsinki, \\ Helsinki, Finland \\ ${ }^{2}$ Department of Oral and Maxillofacial Diseases, Helsinki University Central Hospital (HUCH), \\ Helsinki, Finland \\ ${ }^{3}$ Microbiology Unit of Helsinki University Central Hospital ( $\left.\mathrm{HUCH}\right)$ Laboratory Diagnostics, \\ Helsinki, Finland \\ ${ }^{4}$ Institute of Dentistry, University of Helsinki, Helsinki, Finland
}

\section{INTRODUCTION}

For most people, yeasts are a part of the normal oral flora, and cause infection only if an opportunity appears (Richardson \& Warnock, 2003). In health, the amount of yeast is kept under control by specific and non-specific defence mechanisms of the saliva and the oral mucosa, as well as by competition among oral microbes (Calderone, 2002). Thus, oral candidosis is classically a sign of impaired local or systemic defence mechanisms, or altered oral flora. Reduced saliva secretion due to medication or radiotherapy, primary or secondary deficiencies of humoral or cellmediated immunity, local mucosal diseases, e.g. lichen ruber planus, and the use of wide-spectrum antibiotics, are among well-known predisposing factors.

Candida albicans is the most frequent cause of oral candidosis (in $60-80 \%$ of cases) in patients receiving radiotherapy for head and neck cancer, but at least eight other pathogenic species can be found (Redding et al., 1999; Bagg et al., 2003; Belazi et al., 2004). Although all candidal species cause a similar mucositis, there are remarkable differences in the invasiveness and antifungal susceptibilities among species (Bagg et al., 2003; Belazi et al., 2004). The role of species other than $C$. albicans has become increasingly important, especially in high-risk patients. This underlines the importance of using an appropriate sampling method, in addition to performing accurate microbiological diagnostics, in these patients. Traditional sampling methods for oral yeasts are swabbing the oral mucosa with a cotton swab, scraping with a spatula, suction of saliva, or rinsing the mouth.

The oral cavity goes through radical changes during treatment for oral cancer. Surgical removal of the tumour often results in considerable lack of tissue, and free microvascular flaps of bone, muscle and skin are used for reconstruction. The increase of keratinised surfaces when skin-lined microvascular flaps are used, and subsequent prosthetic rehabilitation with fixed or removable appliances, alter the micro-environment of the oral cavity further. Postoperative radiotherapy to the primary tumour site and regional lymphatics are given to patients with large and aggressive tumours and at risk for metastases. Radiotherapy to a total dose of $50-60$ Gy in 2 Gy daily fractions for 
5-6 weeks starts as soon as the primary healing of the operation wounds has completed, normally 6-8 weeks postoperatively (Saarilahti et al., 2005). For most patients, radiotherapy results in permanent damage to their salivary glands and lifelong xerostomia.

Lack of saliva and development of surfaces more susceptible to heavy yeast colonization cause a lifelong high risk for oral candidosis for these patients. Traditional sampling methods, i.e. saliva culture, mouth rinses or tongue scrapings, are often impossible to perform, and may result in falsenegative results. This is especially problematic, as clinical sympoms may be non-existent due to neural damage and to the considerable decrease in blood flow in the irradiated tissues. The optimal site and method of sampling for oral candidosis in oral cancer patients is not known.

As the first step in a comprehensive follow-up study of the importance of yeast infections in oral cancer patients, we set out to investigate how the sampling method and site of sampling affect the recovery of Candida species from the oral cavity.

\section{METHODS}

Patients and controls. Eighteen previously untreated patients with primary oral cancer (mean age 60 years, range 42-81 years, male:female ratio 11:7) were enrolled in the study (Table 1). All patients were hospitalized due to cancer treatment courses in 2004-2005. Five healthy, non-medicated members of the hospital personnel were included as controls (mean age 42 years, range 28-54 years, male:female ratio $3: 2$ ). For this study, the patients were examined prior to all cancer treatment $(n=5)$, or $2-4$ weeks $(n=5)$ or $8-12$ weeks $(n=8)$ after the primary surgical treatment. Eleven of the 13 patients who had undergone operations $(85 \%)$ had received conventionally fractionated radiotherapy to a mean total dose of 55 Gy (range 20-76 Gy) post-operatively. Two of these $(18 \%)$ had received chemoradiotherapy. The primary sites of the oral cancer were as follows: tongue, six cases; mandible, six cases; maxilla, two cases; buccal mucosa, one case. In three cases, metastasis had been identified. The general status of the dentition, dental status (recorded in the WHO Diseased Missing Filled Index) and oral hygiene (examiner-assessed subjective scale 1-3), as well as the use of antifungals, was recorded.

The study protocol was approved by the ethical committee of the Helsinki University Central Hospital (ethical permit number 525/E6/ $200328 \cdot 01 \cdot 2004)$. All patients signed an informed consent.

Sampling and cultivation of yeasts. Eighteen oral cancer patients and five control subjects were sampled once semi-quantitatively from the labial sulcus, dorsum of the tongue, dental plaque and saliva for cultivation of yeasts. All samples were taken non-invasively with sterile instruments. Particular care was taken to perform the sampling in a standardized way and to avoid contamination from adjacent areas. The precise site of sampling varied a little from patient to patient, depending on the dentate status and anatomical circumstances in the mouth. Saliva samples and samples from the upper labial sulcus were taken with sterile cotton swabs. For the saliva sample, the swab was placed into a moist area in the floor of the mouth for $10 \mathrm{~s}$. For the labial sulcus sample, each sulcus was gently swabbed with single wipes. For the plaque sample, dental plaque of the labial surface of one lower molar tooth was collected with an explorer. Samples from the dorsum of the tongue were taken with one gentle scrape using a spatula.

The samples were collected into sterile tubes containing $0.5 \mathrm{ml}$ sterile saline. The samples were immediately transported to the laboratory,

Table 1. Patient demographics

\begin{tabular}{|c|c|c|c|c|c|}
\hline \multirow[t]{2}{*}{ Characteristic } & \multicolumn{4}{|c|}{ Number of patients } & \multirow[t]{2}{*}{ Number of controls } \\
\hline & Pre-operative & $2-4$ weeks post-operative & 8-12 weeks post-operative & Total & \\
\hline$n$ & 5 & 5 & 8 & 18 & 5 \\
\hline \multicolumn{6}{|l|}{ Sex } \\
\hline Female & 1 & 2 & 4 & $7(39 \%)$ & $2(40 \%)$ \\
\hline Male & 4 & 3 & 4 & $11(61 \%)$ & $3(60 \%)$ \\
\hline \multicolumn{6}{|l|}{ Age (years) } \\
\hline Mean & 61 & 57 & 62 & 60 & 42 \\
\hline Range & - & - & - & $42-81$ & $28-54$ \\
\hline \multicolumn{6}{|l|}{ Location of cancer } \\
\hline Tongue & 1 & 0 & 4 & 5 & 0 \\
\hline Floor of mouth & 1 & 3 & 3 & 7 & 0 \\
\hline Maxilla & 0 & 1 & 1 & 2 & 0 \\
\hline Cheek mucosa & 1 & 0 & 0 & 1 & 0 \\
\hline Metastasis & 2 & 1 & 0 & 3 & 0 \\
\hline \multicolumn{6}{|l|}{ Treatment } \\
\hline Surgery & 0 & 5 & 8 & 13 & 0 \\
\hline Radiotherapy & 0 & 3 & 8 & $11(61 \%)$ & 0 \\
\hline Mean dose & - & - & - & $54 \mathrm{~Gy}$ & - \\
\hline Dose range & - & - & - & 20-76 Gy & - \\
\hline Chemotherapy & 0 & 1 & 1 & $2(11 \%)$ & 0 \\
\hline
\end{tabular}




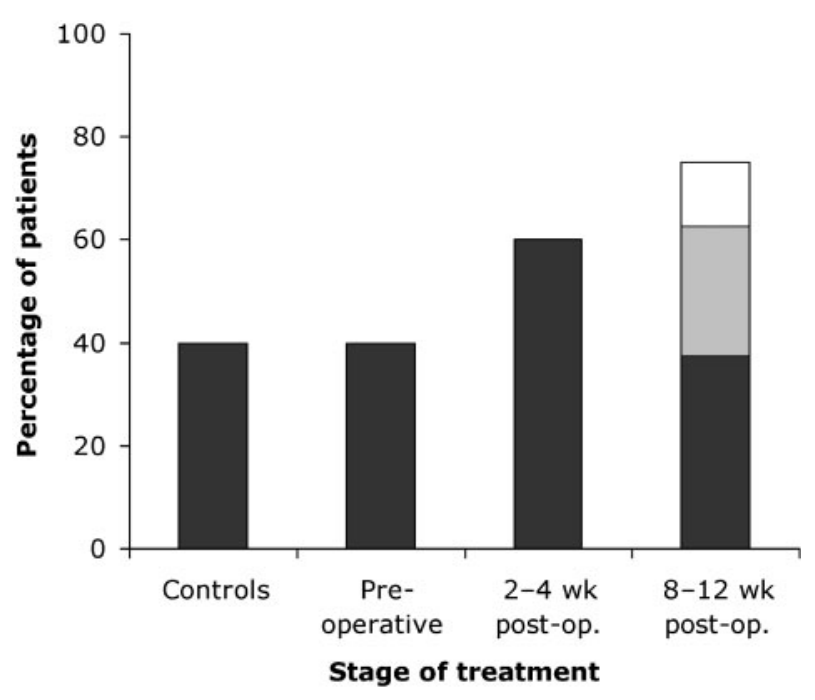

Fig. 1. Incidence of Candida species in oral cancer patients at different stages of treatment, and in control subjects. Black bars, C. albicans; grey bars, non-albicans; white bars, C. albicans + non-albicans; wk, week; post-op., post-operatively.

and $100 \mu \mathrm{l}$ of the saline was plated onto Sabouraud dextrose plates (HUSLAB) after careful mixing, and incubated at $37^{\circ} \mathrm{C}$ for $48 \mathrm{~h}$. Thereafter, colonies were counted and cultivated further on CHROMagar Candida medium (CHROMagar) for the presumptive identification of Candida species. The Bichro-Dubli latex coagglutination test (Fumouze Diagnostics) was used to differentiate between C. albicans and Candida dubliniensis. Species other than $C$. albicans and C. dubliniensis were identified by API 32C auxanographic strips (bioMérieux). Multiple colonies were tested at every identification step.

\section{RESULTS}

The incidence of positive Candida growth in the control and pre-operative groups was $40 \%$ (Fig. 1). After the beginning of cancer treatment, the incidence was found to increase rapidly $(69 \%, 9 / 13$, post-operatively). Of the patients who had undergone operations, $73 \%(6 / 8)$ were positive for Candida 8-12 weeks post-operatively (Table 2). In addition to the increase in the incidence of Candida, the colony

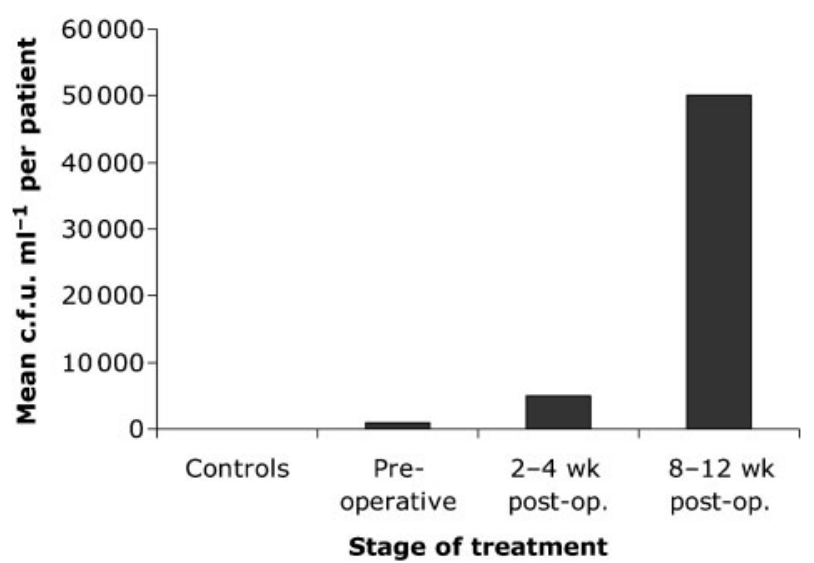

Fig. 2. The density of Candida in colonized oral cancer patients at different stages of treatment, and in control subjects. The values represent the means of the total number of colonies isolated from each patient. Abbreviations: wk, week; post-op., post-operatively.

density also increased after the beginning of the cancer treatment (Fig. 2). In addition, although the incidence of Candida was found to be the same in the control and preoperative groups, the colony density was already found to be markedly higher (15-500-fold) in the oral cancer patient group before the beginning of cancer treatment (Table 3).

Table 2 presents the percentages of samples positive for yeast growth with respect to the site of sampling. The sitespecific samples that were positive most often had been taken from the labial sulcus (100\%) and from the dorsum of the tongue $(91 \%)$. However, the number of c.f.u. was highest in the dental plaque samples. The most sensitive sampling site was found to be the vestibular sulcus, from which all culture-positive cases could be confirmed. In addition, a tongue surface scraping was found to be more sensitive than saliva in detecting Candida in the patients. All sampling methods were equally sensitive in detecting the different species of Candida.

Table 2. Sensitivity of the sampling site in finding subjects positive for Candida

\begin{tabular}{|c|c|c|c|c|c|}
\hline \multirow[t]{2}{*}{ Sampling site } & \multicolumn{4}{|c|}{ Positive patients/total patients (\%) } & \multirow[t]{2}{*}{ Positive controls/total controls (\%) } \\
\hline & Total & Pre-operative & $2-4$ weeks post-operative & 8-12 weeks post-operative & \\
\hline Tooth surface & $8 / 9 \dagger(89)$ & $2 / 2$ & $1 / 1$ & $5 / 6$ & $1 / 2$ \\
\hline Tongue & $10 / 11(91)$ & $2 / 2$ & $2 / 3$ & $6 / 6$ & $1 / 2$ \\
\hline Sulcus & $11 / 11(100)$ & $2 / 2$ & $3 / 3$ & $6 / 6$ & $1 / 2$ \\
\hline
\end{tabular}

${ }^{\star}$ The number of the study subjects positive for Candida as a proportion of the total number of patients sampled.

$\dagger$ The number of subjects positive for Candida at this sampling site as a proportion of the total number of positive subjects. 
Table 3. Colony density of Candida (c.f.u. $\mathrm{ml}^{-1}$ ) at various sampling sites at different stages of treatment for oral cancer, and for control subjects

\begin{tabular}{|lcrrrr|}
\hline \multirow{2}{*}{ Sampling site } & \multicolumn{3}{c|}{ Patients } & \multicolumn{2}{c}{ Controls } \\
\cline { 2 - 5 } & Pre-operative & 2-4 weeks post-operative & 8-12 weeks post-operative & Total \\
\hline Tooth & 730 & 260 & 185240 & 186000 \\
Tongue & 140 & 10015 & 18660 & 10 \\
Sulcus & 380 & 7830 & 30000 & 29000 & 38000 \\
Saliva & 500 & 7035 & 67420 & 75000 \\
\hline
\end{tabular}

Candida albicans was found to be the predominant species. It was the only yeast detected in the control group as well as in the patient groups up to $2-4$ weeks post-operatively (Fig. 1). At 8-12 weeks post-operatively in three of the six Candida-positive patients, a species other than C. albicans strain was identified. Of all the Candida-positive patients who underwent operations, $66 \%$ had Candida albicans, while the rest were species other than $C$. albicans. C. dubliniensis was not found in any of the patient samples. Antifungal prophylaxis, mainly fluconazole $100 \mathrm{mg}$ p.o. or $150 \mathrm{mg}$ i.v. daily, had been given to $44 \%$ of patients. Of the patients with negative yeast growth, $57 \%$ were receiving antifungal treatment. About half of the patients who received antifungal drugs still had positive yeast growth, mainly of species other than C. albicans. Of the patients undergoing radiotherapy, $67 \%$ had positive yeast growth, although five out of eight were receiving antifungal treatment. All patients receiving chemoradiotherapy had positive yeast growth. Of the patients who smoked, $70 \%$ had positive yeast growth.

The general status of the dentition and oral hygiene of the patients was recorded. Of the patients, $56 \%$ were smokers. All patients had been seen by a hospital dentist preoperatively and had no cavities, but had a higher number of missing teeth in the post-operative phase of cancer treatment. Otherwise, the general status of the dentition did not differ markedly between the groups. The oral hygiene of the patients improved during the first months of their cancer treatment.

\section{DISCUSSION}

The sampling of oral cancer patients for candidosis is problematic. The oral cavity goes through radical changes during treatment for oral cancer. Surgical treatment is normally followed by radiotherapy, and occasionally chemoradiotherapy, causing temporary or permanent damage to the salivary glands and a lifelong high risk of oral candidosis for these patients. Traditional sampling methods, i.e. saliva culture, mouth rinses and tongue scrapings, are often impossible to perform, and may result in false-negative results. This is especially problematic, as clinical symptoms may be non-existent due to neural damage and to the considerable decrease of blood flow in the irradiated tissues.
The results of our study showed that the site of sampling for oral yeast cultivation is crucial for a correct diagnosis. Positive growth was most often detected in samples taken from the labial sulcus and upper aspect of the tongue. Dental plaque on tooth surfaces was found to have the highest density of Candida colonization of all sites sampled. It was thus found to be the most significant source of Candida infection, which emphasizes the role of dental care in these patients. However, there is a risk of overdiagnosis and treatment when sampling the tooth surfaces, as the amount of yeast cultured is used for treatment decisions in these patients. The labial sulci and dorsum of the tongue are often more relevant sampling sites in oral cancer patients, in whom surgical operations may have deformed other anatomical areas of the oral cavity. The optimal sampling site for Candida was found to be the vestibular sulcus.

Our findings of an increase in the incidence of Candida and a shift from C. albicans to other Candida species is in accordance with the findings of others (Bagg et al., 2003; Redding et al., 2004). These findings have been thought to be a result of the disease and its treatment, and of the use of azole antifungals. Our findings confirm that the choice of antifungals and the mode of drug administration need careful attention in these patients. The use of systemic antifungals to control superficial infection at a site of decreased blood flow and lack of saliva results in suboptimal drug concentrations and a high risk of the development and selection of resistant strains. The use of systemic drugs cannot be sanctioned unless the patient is unable to apply topical drugs or is immunocompromised.

\section{ACKNOWLEDGEMENTS}

This work was in part supported by grant TYH 3245 of the Helsinki University Central Hospital. J. H. M. has been supported by the Ulf Nilsonne Foundation (SalusAnsvar Prize), Stockholm, Sweden. This study was also supported by a Finnish Dental Association Apollonia student grant.

\section{REFERENCES}

Bagg, J., Sweeney, M. P., Lewis, M. A. O., Jackson, M. S., Coleman, D., Al Mosaid, A., Baxter, W., McEndrick, S. \& McHugh, S. (2003). High prevalence of non-albicans yeasts and detection of anti-fungal resistance in the oral flora of patients with advanced cancer. Palliat Med 17, 477-481. 
Belazi, M., Velegraki, A., Koussidou-Eremondi, T., Andreadis, D., Hini, S., Arsenis, G., Eliopoulou, C., Destouni, E. \& Antoniades, D. (2004). Oral Candida isolates in patients undergoing radiotherapy for head and neck cancer: prevalence, azole susceptibility profiles and response to antifungal treatment. Oral Microbiol Immunol 19, 347-351.

Calderone, R. (2002). Candida and Candidiasis. Washington, DC: American Society for Microbiology.

Redding, S. W., Zellars, R. C., Kirkpatrick, W. R. \& 7 other authors (1999). Epidemiology of oropharyngeal Candida colonization and infection in patients receiving radiation for head and neck cancer. J Clin Microbiol 37, 3896-3900.
Redding, S. W., Dahiya, M. C., Kirkpatrick, W. R., Coco, B. J., Patterson, T. F., Fothergill, A. W., Rinaldi, M. G. \& Thomas, C. R., Jr (2004). Candida glabrata is an emerging cause of oropharyngeal candidiasis in patients receiving radiation for head and neck cancer. Oral Surg Oral Med Oral Pathol Oral Radiol Endod 97, $47-52$.

Richardson, M. D. \& Warnock, D. W. (2003). Fungal Infection; Diagnosis and Management. Oxford: Blackwell Publishing.

Saarilahti, K., Kouri, M., Collan, J., Hämäläinen, T., Atula, T., Joensuu, H. \& Tenhunen, M. (2005). Intensity modulated radiotherapy for head and neck cancer: evidence for preserved salivary gland function. Radiother Oncol 74, 251-258. 